\title{
Serviços de alimentação comercial: fator de risco para a saúde pública?
}

\author{
Commercial food services: risk factor for public health?
}

Maria Regina Sarkis Peixoto JOELE ${ }^{1}$ Consuelo Lima SOUSA ${ }^{2 \star}$, Lúcia de Fátima Henriques LOURENÇO²

*Endereço para correspondência: ${ }^{2}$ Laboratório de Microbiologia de Alimentos, Faculdade de Engenharia de Alimentos, Instituto de Tecnologia, Universidade Federal do Pará, Avenida Perimetral s/n, Guamá, Belém, PA, Brasil. CEP 6075-110, Caixa postal 479. Telefone: 91 3201-1293, Fax: 91 3201-7456. E-mail: sousa@ufpa.br ${ }^{1}$ Instituto Federal de Educação, Ciência e Tecnologia do Pará, campus Castanhal. Castanhal, Pará, Brasil. Recebido: 06.06.2013 - Aceito para publicação: 17.03.2014

\section{RESUMO}

Neste trabalho foram avaliadas as condições higiênico-sanitárias de restaurantes comerciais com serviços do tipo self-service localizados na cidade de Belém/PA. No primeiro semestre de 2012, foram avaliados os procedimentos adotados quanto às Boas Práticas de Fabricação em 10 estabelecimentos tipo self-service. A investigação foi efetuada com auxílio da lista de verificação proposta na Resolução RDC no 216/2004 da Agência Nacional de Vigilância Sanitária (ANVISA) do Ministério da Saúde, que classifica os serviços de alimentação de acordo com o porcentual de atendimento dos itens em: Grupo 1 - BOM (76 a 100 \% de atendimento dos itens), Grupo 2 - REGULAR ( 51 a 75 \% de atendimento dos itens) e Grupo 3 - RUIM ( 0 a $50 \%$ de atendimento dos itens). Dos restaurantes avaliados, $20 \%$ foram classificados no grupo 3 (RUIM), $30 \%$ no grupo 2 (REGULAR) e $50 \%$ no grupo $1(\mathrm{BOM})$. De modo geral, os restaurantes avaliados já estavam em processo de implantação das Boas Práticas, porém com aspectos a serem corrigidos. Entre os principais problemas dos estabelecimentos avaliados destacam-se o fluxo cruzado em função de estrutura física do local, a higienização inadequada de utensílios e de equipamentos, e a falta de monitoramento por parte do responsável pelas atividades do local.

Palavras-chave. restaurantes, higiene dos alimentos, manipulação de alimentos.

\begin{abstract}
This investigation aimed at evaluating the hygienic and sanitary conditions in self-service type restaurants located in Belém, PA. The procedures related to the Good Manufacturing Practices were evaluated in 10 self-service restaurants, during the first semester of 2012. For conducting the study, the checklist proposed in the Resolution RDC no. 216/2004 of the National Sanitary Surveillance Agency - Ministry of Health. This RDC classifies the food services according to the percentage of attendance of the items in: group 1 - GOOD (76 to $100 \%$ of items attendance), group 2 - REGULAR (51 to $75 \%$ of items attendance) and group 3 - BAD ( 0 to $50 \%$ of items attendance). Of the evaluated restaurants, $20 \%$ were classified into group 3 (BAD), $30 \%$ into group 2 (REGULAR) and $50 \%$ into group 1 (GOOD). In general, the restaurants were already in the process of the Good Practices implementation, but being in a stage for correcting some issues. Among the main problems detected in the evaluated restaurants, the following questions were evidenced: the cross-flow due to the place physical structure, the inadequate cleaning of utensils and equipment, and the lack of monitoring by the manager in charge of the place activities.
\end{abstract}

Keywords. restaurants, food hygiene, food manipulation. 
Peixoto MRSJ, Sousa CL, Lourenço LFH. Serviços de alimentação comercial: fator de risco para a saúde pública?. Rev Inst Adolfo Lutz. São Paulo, 2014; 73(1):113-18.

\section{INTRODUÇÃO}

As mudanças socioeconômicas ocorridas nos últimos anos, como urbanização, industrialização, a distância entre o domicílio e o local de trabalho, entre outros fatores, contribuíram para o número cada vez maior de pessoas que se alimentam fora de casa ${ }^{1}$. Os Serviços de Alimentação têm por objetivo oferecer uma alimentação de qualidade fora de domicílio e que seja adequada às necessidades do consumidor levando em consideração seus hábitos e preferências².

Nos últimos anos, houve aumento significativo no número de restaurantes no Brasil, onde em 2013, o setor de alimentação coletiva forneceu 11,7 milhões de refeições/ dia com faturamento 16,6 bilhões de reais, com tendência de crescimento nos próximos anos ${ }^{3}$. Entretanto, observase que, paralelamente ao crescimento deste segmento, há aumento da ocorrência de Doenças Veiculadas por Alimentos (DVAs) decorrente da ausência de controle rigoroso no processamento, no armazenamento e na distribuição dos alimentos ${ }^{4}$.

Dentre os fatores de prevenção de doenças, destacase a qualidade da matéria prima, higiene dos manipuladores e dos utensílios utilizados, assim como o monitoramento do tempo e temperatura das preparações alimentares. Portanto, a detecção e rápida correção das falhas no processamento, bem como a adoção de medidas preventivas, são as principais estratégias para o controle de qualidade ${ }^{5}$.

Para garantir a qualidade do produto e a saúde do consumidor é necessário que todos os estabelecimentos que produzem e comercializam alimentos adotem ferramentas de gestão de qualidade como as Boas Práticas de Fabricação (BPF), que correspondem a conjunto de princípios e regras para o correto manuseio de alimentos e que tem como meta principal a redução dos riscos, além de ser um sistema de baixo custo, eficaz e de fácil execução ${ }^{6}$.

Os Serviços de Alimentação que são devidamente organizados e ordenados dentro dos princípios estabelecidos pelas BPF, permitem a identificação de falhas operacionais ou administrativas que possam ocorrer durante o processo produtivo, garantindo também a melhoria na qualidade e minimizando os riscos de surtos de origem alimentar ${ }^{7}$.

Devido à importância do controle na elaboração de alimentos que atendam às características e integridade do produto, bem como a saúde dos consumidores, o objetivo do presente trabalho foi avaliar as condições higiênico-sanitárias de restaurantes comerciais com serviços do tipo self-service localizados na cidade de Belém/PA.

\section{MATERIAL E MÉTODOS}

Foram avaliados, no período compreendido entre janeiro a julho de 2012, $40 \%$ dos restaurantes $(n=10)$ tipo self-service, com produção média de 200 refeições diárias, localizados no centro da cidade de Belém/Pará e que aceitaram participar da presente pesquisa. Os restaurantes foram identificados por letras de A a J.

Para avaliação das BPF, foi utilizada a lista de verificação proposta na Resolução RDC no 216/2004 , constituída por 129 itens de verificação agrupados em 12 blocos por assunto, sendo: Bloco 1 - "Edificações, Instalações, Equipamentos, Móveis e Utensílios"; Bloco 2 - "Higienização de Instalações, Equipamentos, Móveis e Utensílios"; Bloco 3 - "Controle Integrado de Vetores e Pragas Urbanas"; Bloco 4 - "Abastecimento de Água"; Bloco 5 - "Manejo de Resíduos"; Bloco 6 - "Manipuladores"; Bloco 7 - "Matérias-primas, Ingredientes e Embalagens"; Bloco 8 - "Preparação do Alimento"; Bloco 9 - "Armazenamento e Transporte do Alimento Preparado"; Bloco 10 "Exposição ao Consumo do Alimento Preparado"; Bloco 11 - "Documentação e Registro e Bloco"; 12 "Responsabilidade", permitindo sua classificação em grupos, de acordo com o atendimento a esses itens.

Os itens foram classificados de acordo com os critérios: Conforme (C) quando estavam em conformidade com a legislação sanitária vigente; Não Conforme (NC) quando não estavam em conformidade e Não se Aplica (NA) para os itens que não tinham relevância. Cada bloco da ficha de avaliação obteve um valor, em porcentagem, em função da conformidade ou não conformidade dos itens. Todos os itens atendidos foram somados para obter a porcentagem geral do estabelecimento. A classificação geral do estabelecimento foi feita de acordo com o estabelecido na Resolução RDC n ${ }^{\circ}$ 216/2004\%: Grupo 1 - BOM (76 a $100 \%$ de atendimento dos itens), Grupo 2 - REGULAR (51 a $75 \%$ de atendimento dos itens) e Grupo 3 - RUIM (0 a 50 \% de atendimento dos itens).

Nas vistorias para o preenchimento da lista, foi acompanhada a rotina de trabalho de cada restaurante durante três dias consecutivos através de observação visual, verificação de documentação e informações fornecidas por funcionários e proprietários. Após cada vistoria foi realizada uma reunião com o responsável técnico 
Peixoto MRSJ, Sousa CL, Lourenço LFH. Serviços de alimentação comercial: fator de risco para a saúde pública?. Rev Inst Adolfo Lutz. São Paulo, 2014; 73(1):113-18.

e ajudantes onde foi repassado o plano de ações corretivas, onde estavam descritas todas as não conformidades detectadas e as correções que deveriam ser feitas, também especificando o responsável e o prazo de execução.

\section{RESULTADOS E DISCUSSÃO}

De acordo com o percentual de conformidades geral dos restaurantes (Figura 1), verificou-se que $20 \%$ foram classificados no grupo 3 (RUIM), $30 \%$ no grupo 2 (REGULAR) e $50 \%$ no grupo 1 (BOM). É recomendado para os serviços de alimentação que os atributos avaliados tenham porcentagens de conformidades maiores que $70 \%$

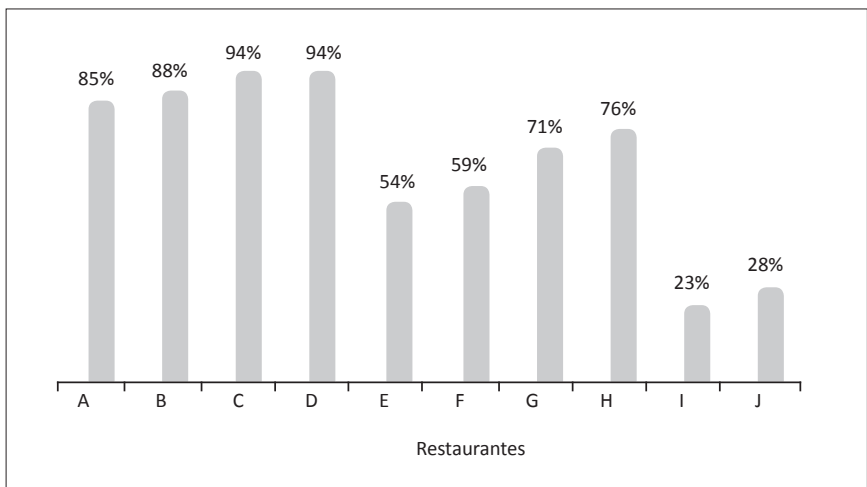

Figura 1. Porcentagem geral de atendimento aos atributos da lista de verificação das Boas Práticas de cada restaurante avaliado. para garantir a produção de alimentos seguros?.

Tabela 1. Percentual de conformidade de cada Bloco da lista de verificação das BPF por restaurante avaliado, localizados na cidade do Belém PA, no período de Janeiro a Julho de 2012

\begin{tabular}{|c|c|c|c|c|c|c|c|c|c|c|}
\hline \multirow{2}{*}{ Blocos } & \multicolumn{10}{|c|}{ Restaurantes (\% de conformidades) } \\
\hline & A & B & $\mathrm{C}$ & D & $\mathbf{E}$ & $\mathbf{F}$ & G & $\mathbf{H}$ & I & $\mathbf{J}$ \\
\hline Bloco 1 & 52 & 59 & 86 & 90 & 44 & 59 & 75 & 62 & 19 & 30 \\
\hline Bloco 2 & 77 & 100 & 100 & 100 & 54 & 85 & 85 & 92 & 38 & 34 \\
\hline Bloco 3 & 80 & 100 & 100 & 100 & 60 & 60 & 80 & 60 & 0 & 0 \\
\hline Bloco 4 & 72 & 100 & 100 & 100 & 100 & 100 & 100 & 100 & 100 & 100 \\
\hline Bloco 5 & 67 & 100 & 100 & 100 & 67 & 100 & 67 & 67 & 67 & 42 \\
\hline Bloco 6 & 67 & 92 & 86 & 86 & 73 & 82 & 82 & 82 & 23 & 44 \\
\hline Bloco 7 & 70 & 95 & 75 & 95 & 91 & 55 & 91 & 91 & 54 & 21 \\
\hline Bloco 8 & 35 & 95 & 95 & 90 & 57 & 54 & 71 & 64 & 21 & 28 \\
\hline Bloco 9 & 50 & 67 & NA & NA & NA & NA & NA & NA & NA & 37 \\
\hline Bloco 10 & 67 & 91 & 92 & 92 & 60 & 60 & 80 & 80 & 40 & 43 \\
\hline Bloco 11 & 50 & 50 & 100 & 50 & 0 & 0 & 0 & 50 & 0 & 0 \\
\hline Bloco 12 & 100 & 100 & 100 & 100 & 100 & 100 & 100 & 100 & 100 & 0 \\
\hline
\end{tabular}

Bloco 1. Edificação, instalação, equipamentos moveis e utensílios; Bloco 2. Higienização de Instalações, equipamentos, móveis e utensílios; Bloco 3. Controle Integrado de Vetores e Pragas urbanas; Bloco 4. Abastecimento de água; Bloco 5. Manejo de Resíduos; Bloco 6. Manipuladores; Bloco 7. Matérias primas, ingredientes e embalagens; Bloco 8. Preparação do Alimento; Bloco 9. Armazenamento e transporte do alimento preparado; Bloco 10. Exposição ao consumo do alimento preparado; Bloco 11. Documentação e registro; Bloco 12. Responsabilidade. NA = Não se aplica 
Peixoto MRSJ, Sousa CL, Lourenço LFH. Serviços de alimentação comercial: fator de risco para a saúde pública?. Rev Inst Adolfo Lutz. São Paulo, 2014; 73(1):113-18.

Saccol et $\mathrm{al}^{6}$ verificaram, por meio de uma lista de verificação das BPF, que $83 \%$ dos estabelecimentos (restaurantes, padarias e confeitarias) apresentaram classificação RUIM na primeira aplicação da lista, entretanto após a utilização do plano de ação corretiva proposto, houve um decréscimo considerável dos estabelecimentos classificados no grupo 3.

Os percentuais de conformidade de cada Bloco da lista de verificação das BPF dos 10 restaurantes avaliados encontram-se na Tabela 1.

Alguns dos problemas encontrados durante a avaliação nos restaurantes estavam relacionados com as edificações e instalações (Bloco 1), que não foram projetadas de forma a possibilitar um fluxo ordenado e sem cruzamentos entre as etapas de preparação do alimento, não havia separação física entre as atividades e na maioria dos estabelecimentos, o dimensionamento das instalações não era adequado ao número de refeições produzidas. A adequação da edificação e instalações constitui uma condição para a implementação das $\mathrm{BPF}^{2}$.

Em 70 \% dos estabelecimentos, as instalações e os equipamentos não estavam mantidos em condições higiênico-sanitárias apropriadas (Bloco 2), devido principalmente à frequência inadequada das operações de higienização, realizadas somente no final do expediente, que ocasionavam acúmulo de resíduos. $\mathrm{Na}$ maioria dos estabelecimentos, não havia local adequado para armazenar os utensílios utilizados na higienização, não sendo encontrado também registro destas operações. Equipamentos e utensílios com higienização deficiente têm sido responsáveis, isoladamente ou associados a outros fatores, por surtos de doenças de origem alimentar ou por alterações de alimentos processados ${ }^{10}$.

Entre os restaurantes avaliados, dois não apresentavam qualquer tipo de controle de pragas (Bloco 3) e em três, apesar da aplicação de controle químico foi detectada a presença de insetos (moscas e baratas) na área de produção, o que segundo Cruz et $\mathrm{al}^{11}$ pode acarretar prejuízos para a segurança microbiológica dos alimentos. Quanto à qualidade da água (Bloco 4), verificou-se que todos os restaurantes utilizavam água potável, proveniente da rede pública de abastecimento, na preparação dos alimentos.

Em relação ao acondicionamento dos resíduos (Bloco 5), 60 \% dos restaurantes não possuíam recipientes adequados, em número e capacidade suficientes. Em um dos restaurantes, foi observada a deposição de resíduos em área aberta até a coleta urbana. Segundo Miranda et $\mathrm{al}^{12}$, recipientes sem acionamento automático podem contribuir para o aumento do risco de contaminação de alimentos, sendo que na maioria das vezes a não aquisição das mesmas está relacionada a falta de conhecimento dos responsáveis sobre a importância da exigência legal.

Foi constatado que, nos restaurantes avaliados, os manipuladores (Bloco 6) não higienizavam as mãos corretamente, devido principalmente à ausência de produtos adequados e também não foram observados cartazes com orientação aos manipuladores sobre a correta higienização das mãos, em locais de fácil visualização. Em relação à capacitação dos manipuladores, somente $60 \%$ dos estabelecimentos apresentaram registro de realização do curso de capacitação e/ou entrega de certificados de manipulador de alimentos emitidos pelo órgão competente. $\mathrm{Na}$ maioria dos restaurantes (70 \%), os manipuladores utilizavam uniformes completos, limpos e conservados, com unhas curtas e sem esmaltes, sem adornos pessoais e maquiagem. Em $20 \%$ dos restaurantes, funcionários com lesões cutâneas principalmente nas mãos, sem devida proteção, não eram afastados da atividade de preparação de alimentos. Estudos realizados em diversos estados do Brasil constataram que a frequência da higienização das mãos dos manipuladores de alimento era inadequada e, além disso, foi observado o uso de adornos (especialmente anéis, relógios e pulseiras) e esmalte nas unhas por esses profissionais durante o trabalho ${ }^{13}$.

$\mathrm{O}$ recebimento de matérias-primas e ingredientes (Bloco 7) é a primeira etapa de controle higiênicosanitário no estabelecimento e deve compreender atividades de conferência da qualidade dos produtos recebidos $^{12}$. Entre os estabelecimentos avaliados, $30 \%$ não faziam seleção de fornecedores e, em apenas um desses foi detectada a presença de alimentos sem procedência (sem identificação do fornecedor, data de fabricação e prazo de validade). Nenhum dos restaurantes avaliados realizava controle de temperatura das matérias-primas no recebimento e armazenamento, assim como identificação nos alimentos a serem congelados. O descaso com a temperatura das matérias-primas perecíveis é uma das principais inadequações relatadas durante avaliações em serviços de alimentação, que pode levar ao desperdício e principalmente, causar risco à saúde do consumidor ${ }^{2}$. No restaurante J foi observado que os equipamentos utilizados para armazenar os alimentos encontravam-se em mal estado de conservação e higiene com a utilização de palets e prateleiras de madeira, o que também contribuiu para a 
Peixoto MRSJ, Sousa CL, Lourenço LFH. Serviços de alimentação comercial: fator de risco para a saúde pública?. Rev Inst Adolfo Lutz. São Paulo, 2014; 73(1):113-18.

baixo índice de conformidade obtido e que vai contra ao preconizado na legislação vigente brasileira.

$\mathrm{Na}$ etapa de preparo dos alimentos (Bloco 8), verificou-se que não estavam sendo adotadas medidas para minimizar o risco de contaminação cruzada em $70 \%$ das unidades, em decorrência da ausência de separação física entre as atividades, possibilitando o contato direto entre alimentos crus e cozidos. Em $100 \%$ das unidades, o acondicionamento de matérias-primas e ingredientes, que não foram totalmente utilizados, estava inadequado, sem identificação ou mantido na embalagem original, o que não é recomendado pela legislação ${ }^{8}$. Outro item encontrado totalmente não conforme foi o controle da temperatura, pois nenhum dos estabelecimentos avaliados realizava este controle durante o preparo (tratamento térmico) e nas preparações alimentares expostas ao consumo. Um dos pontos críticos relevante nas BPF corresponde ao binômio tempo e temperatura, uma vez que a permanência de alimentos nas temperaturas consideradas como zona de perigo $\left(10^{\circ} \mathrm{C}\right.$ a $\left.60^{\circ} \mathrm{C}\right)$ promove a multiplicação rápida de micro-organismos, os quais poderão ocasionar danos à saúde do consumidor ${ }^{14}$. Constatou-se também que 50 $\%$ dos restaurantes realizavam o descongelamento dos alimentos de forma inadequada, sempre em temperatura ambiente com o produto fora da embalagem.

Dos dez restaurantes avaliados apenas três armazenavam e transportavam alimentos preparados (Bloco 9), entretanto de maneira inadequada, pois os mesmos estavam sem identificação (data de preparo e prazo de validade) e enquanto aguardavam o transporte, eram acondicionados juntamente com matérias-primas nas câmaras de refrigeração ou em temperatura ambiente. Os veículos utilizados para o transporte de refeições prontas para o consumo não eram exclusivos para este fim, algumas vezes utilizados também para transportar outras cargas (material de limpeza). O transporte do alimento preparado deve ocorrer em condições adequadas de tempo e temperatura e em veículos dotados de cobertura para proteção da carga, não devendo transportar outras cargas que comprometam a qualidade higiênico-sanitária do alimento preparado ${ }^{7}$.

Dentre os restaurantes avaliados, dois não possuíam balcões expositores (E e J) e em somente dois $(\mathrm{C}$ e $\mathrm{D})$ os balcões tinham barreira de proteção contra a contaminação do alimento em decorrência da proximidade ou da ação do consumidor. O principal objetivo dos balcões térmicos é fornecer produtos em temperatura sensorialmente agradável e mantê-los em condição de tempo e temperatura seguros $\left(\mathrm{t}>60^{\circ} \mathrm{C}\right.$ por no máximo 6 horas) sob o ponto de vista microbiológico ${ }^{7,13}$. Nenhum dos restaurantes fazia o controle de temperatura dos alimentos e equipamentos. Quanto à distribuição dos alimentos prontos que necessitavam de regrigeração, verificou-se que apenas $50 \%$ dos estabelecimentos possuíam balcão refrigerado, sendo que nos demais, os alimentos estavam expostos à temperatura ambiente, não sendo tomada nenhuma medida de controle a fim de evitar crescimento microbiano. Em relação ao acondicionamento dos utensílios, constatou-se que, em um dos restaurantes, estes estavam sendo acondicionados em local inadequado e sem proteção.

Quanto aos registros e documentação dos restaurantes (Bloco 11), apenas $50 \%$ tinham Manual de Boas Práticas (MBP) e somente um apresentou os Procedimentos Operacionais Padronizados (POP) com seus respectivos registros. A falta de documentação é uma das irregularidades mais comuns, relatadas em auditorias de $\mathrm{BPF}$ em serviços de alimentação ${ }^{1,5}$. Em relação à responsabilidade técnica (Bloco 12), verificou-se que $90 \%$ dos restaurantes tinham em seu quadro de pessoal um profissional da área de alimentos (nutricionista).

Ebone et $\mathrm{al}^{15}$, ao analisarem estratégias utilizadas para a gestão da qualidade higiênico-sanitária em unidades produtoras de refeições comerciais de Florianópolis (SC), detectaram que as unidades necessitavam de melhorias para oferecer alimentos mais seguros, sendo imprescindível a conscientização dos proprietários e dos gerentes sobre a importância de servir alimentos de qualidade que não causem danos à saúde do comensal.

Ferreira et $\mathrm{al}^{16}$, ao avaliarem Unidades de Alimentação e Nutrição (UAN) de pequeno e médio portes localizadas na região metropolitana de Belo Horizonte, concluíram que apesar da boa classificação geral das UAN, foram detectadas falhas nos blocos "edificação e instalações" e "equipamentos, móveis e utensílios".

\section{CONCLUSÃO}

Entre os principais problemas dos estabelecimentos avaliados destacaram-se o fluxo cruzado durante o processo produtivo devido às falhas estruturais nas edificações, a higienização inadequada de utensílios e equipamentos e a falta de monitoramento por parte do responsável pelas atividades do local, fatores importantes para a definição da qualidade do ambiente 
em que são produzidos alimentos para o consumo. $\mathrm{Na}$ maioria dos restaurantes avaliados (60\%), foi observado que, mesmo que parcialmente, já estavam sendo aplicados procedimentos de Boas Práticas, porém ainda com deficiências, que podem ser minimizadas com adoção de treinamentos e supervisão contínuos. A adoção efetiva das Boas Práticas deve ser o desafio de todo o pessoal envolvido na manipulação de alimentos, visando à produção de alimentos seguros e com qualidade aos consumidores, sendo, portanto necessário que todas as não conformidades apontadas sejam corrigidas e que procedimentos de manipulação e higiene sejam adotados de acordo com o Plano de Ação encaminhado aos restaurantes.

\section{REFERÊNCIAS}

1. Mello AG, Back FS, Colares LGT. Condições higiênico-sanitárias de restaurantes self-services localizados no estado do Rio de Janeiro. Hig Alim.2011;25(2):64-9.

2. Medeiros L, Dall'AgnolII LP, Botton AS, Smaniotto H, Potter $\mathrm{R}$, Campos MMA, et al. Qualidade higiênico-sanitária dos restaurantes cadastrados na Vigilância Sanitária de Santa Maria, RS, Brasil, no período de2006 a 2010. Ciênc Rural.2013;43(1):81-6. doi.org/10.1590/S0103-84782012005000146

3. ABERC - Associação Brasileira das Empresas de Refeições Coletivas. Mercado real. Estimativa 2014. [acesso 2014 Jan 24]. Disponível em: [http://www.aberc.com.br/mercadoreal.asp]

4. Organização Mundial de Saúde. Food borne disease: a focus for health education. 695 Geneva: World Health Organization. 2000;198p.

5. Seixas FRF, Seixas JRF, Reis JA, Hoffmann FL. Check-list para diagnóstico inicial das Boas Práticas de Fabricação (BPF) em estabelecimentos produtores de alimentos da cidade de São José do Rio Preto (SP). Rev Analytica.2008;33:12-7.

6. Saccol ALF, Stangarlin L, Richards NS, Hecktheuer LH. Avaliação das boas práticas em duas visões: técnica e da empresa. Braz J Food Technol.2009; 1:19-23.
7. Fonseca MP, Manfridini LA, José JFBO, Tomazini APB, Martini HSD, Ribeiro RCL, et al. Avaliação das condições físicofuncionais de restaurantes comerciais para implementação das boas práticas. Alim Nutr.2010;21(2):251-7.

8. BRASIL. Ministério da Saúde. Agência Nacional de Vigilância Sanitária. Resolução - RDC no. 216, de 15 de setembro de 2004. Dispõe sobre Regulamento Técnico de Boas Práticas para Serviços de Alimentação. Diário Oficial [da] União. Brasília, 2004. [acesso 2014 Jan 20]. Disponível em: [http://www.anvisa.gov.br].

9. Sousa CL, Neves ECA, Lourenço LFH, Costa EB, Monteiro RRC. Microbiological and hygienic-sanitary conditions diagnostic in light frozen food industry in Belém/PA. Alim Nutr.2009;20(3):375-81.

10. Mezzari MF, Ribeiro AB. Avaliação das condições higiênicosanitárias da cozinha de uma escola municipal de Campo Mourão - Paraná. SaBios Rev Saúde Biol.2012;7(3):60-6.

11. CruzAG,CenciAS,MaiaMCA.Pré-requisitosparaimplementação do sistema APPCC em uma linha de alface minimamente processada. Ciênc Tecnol Aliment.2006;26(1):104-9. doi. org/10.1590/S0101-20612006000100018

12. Miranda ACB, Baião RCL. Avaliação das boas práticas na fabricação de preparações à base de pescados crus em restaurante japonês. C\&D Rev Eletr Fainor.2011;4(1):52-61.

13. Cardoso RCV, Souza EVA, Santos PQ. Unidades de alimentação e nutrição nos campi da Universidade Federal da Bahia: um estudo sob a perspectiva do alimento seguro. Rev Nutr.2005;18(5):66980. doi.org/10.1590/S1415-52732005000500010

14. Storck CR, Dias MAMF. Monitoramento da temperatura de preparações quentes e frias em restaurantes self-services na zona urbana de Santa Maria. Nutr Pauta.2003;11(59):31- 5.

15. Ebone MV, Cavalli SB, Lopes SJ. Segurança e qualidade higiênico-sanitária em unidades produtoras de refeições comerciais. Rev Nutr.2011;24(5):725-34. doi.org/10.1590/S141552732011000500006.

16. Ferreira MA, São José JFB, Tomazini APB, Martini HSD, Milagres RCM, Pinheiro-Sant'Ana HM. Avaliação da adequação às boas práticas em unidades de alimentação e nutrição. Rev Inst Adolfo Lutz.2011;70(2): 230-5. 